\title{
CTLA4 blockade and GM-CSF combination immunotherapy alters the intratumor balance of effector and regulatory $T$ cells
}

\author{
Sergio A. Quezada, Karl S. Peggs, Michael A. Curran, and James P. Allison \\ Howard Hughes Medical Institute, Department of Immunology, Memorial Sloan-Kettering Cancer Center, New York, New York, USA.
}

\begin{abstract}
CTL-associated antigen 4 (CTLA4) blockade releases inhibitory controls on $\mathrm{T}$ cell activation and proliferation, inducing antitumor immunity in both preclinical and early clinical trials. We examined the mechanisms of action of anti-CTLA4 and a GM-CSF-transduced tumor cell vaccine (Gvax) and their impact on the balance of effector T cells (Teffs) and Tregs in an in vivo model of B16/BL6 melanoma. Tumor challenge increased the frequency of Tregs in lymph nodes, and untreated tumors became infiltrated by $\mathrm{CD}^{+} \mathrm{Foxp3}^{-}$and $\mathrm{CD}^{+} \mathrm{Foxp}^{+}$ $T$ cells but few $\mathrm{CD8}^{+} \mathrm{T}$ cells. Anti-CTLA4 did not deplete Tregs or permanently impair their function but acted in a cell-intrinsic manner on both Tregs and Teffs, allowing them to expand, most likely in response to self antigen. While Gvax primed the tumor-reactive Teff compartment, inducing activation, tumor infiltration, and a delay in tumor growth, the combination with CTLA4 blockade induced greater infiltration and a striking change in the intratumor balance of Tregs and Teffs that directly correlated with tumor rejection. The data suggest that Tregs control both $\mathrm{CD}^{+}$and $\mathrm{CD8}^{+} \mathrm{T}$ cell activity within the tumor, highlight the importance of the intratumor ratio of effectors to regulators, and demonstrate inversion of the ratio and correlation with tumor rejection during Gvax/anti-CTLA4 immunotherapy.
\end{abstract}

\section{Introduction}

Devising ways to mobilize antitumor $T$ cells with effector activity is one of the main goals of modern immunotherapy. Advances in basic immunology are leading us toward a more complete understanding of the mechanisms that regulate immune responses and particularly the checkpoints that limit the initiation or the magnitude of these responses in order to prevent autoimmunity and that shape immune responses to tumors. The early stages of $\mathrm{T}$ cell activation are regulated by interaction of B7-1 and B7-2, whose expression is sharply restricted to DCs and other professional APCs, with their counterreceptors CD28 and CTLassociated antigen 4 (CTLA4) on the T cell surface (1). CD28 is constitutively expressed and provides costimulatory signals that are critical for proliferation of naive T cells, while CTLA4 is transiently expressed after activation and plays a critical role in downregulating $\mathrm{T}$ cell responses. CD28 ligation has been shown to enhance $T$ cell proliferation by inducing production of IL-2 and antiapoptotic factors. CTLA4 ligation, on the other hand, has been shown to inhibit IL-2 production and the production of cyclins, cyclin-dependent kinases, and other components of the machinery needed for cell cycle progression. The result is that CTLA4 engagement limits proliferation of activated T cells (2-4). We have shown that localization of CTLA4 to the immune synapse is directly proportional to TCR signal strength, an observation suggesting that high-affinity interactions might be more strongly regulated by CTLA4 than weak interactions $(5,6)$. Together, these results suggest that there is a dynamic balance

Nonstandard abbreviations used: CTLA4, CTL-associated antigen 4; Foxp3, forkhead box protein P3; i.d., intradermally; Teff, effector T cell; TIL, tumor-infiltrating lymphocyte.

Conflict of interest: The authors have declared that no conflict of interest exists. Citation for this article: J. Clin. Invest. 116:1935-1945 (2006). doi:10.1172/JCI27745 among TCR, CD28, and CTLA4 signaling that determines the outcome of a T cell's encounter with an APC; that CTLA4 can serve to limit the magnitude of the response by differential inhibition of T cell proliferation; and that blockade of this inhibition could increase the magnitude of $\mathrm{T}$ cell responses.

Blockade of CTLA4 with anti-CTLA4 antibodies can induce rejection of several types of established transplantable tumors in mice, including colon carcinoma, fibrosarcoma, prostatic carcinoma, lymphoma, and renal carcinoma (7-11). Despite its effectiveness in these experimental tumor models, CTLA4 blockade failed to induce rejection of less immunogenic tumors such as the B16 melanoma and SM1 mammary carcinoma $(12,13)$. For less immunogenic tumors, combinatorial therapies may be required in order to generate similar therapeutic outcomes. We previously demonstrated that CTLA4 blockade in combination with a vaccine consisting of irradiated tumor transduced to express the cytokine GM-CSF (Gvax) could eradicate established B16 melanoma (12, 14) and SM1 mammary carcinoma (13). Interestingly, Gvax monotherapy is effective at inducing prophylactic immunity to B16 melanoma by increasing cross-priming of naive $\mathrm{CD}^{+} \mathrm{T}$ cells by host $\operatorname{APCs}(15,16)$, but it fails when used as a monotherapy for established tumors (12). Together these studies demonstrate that CTLA4 blockade induces rejection of several tumor types in different mouse strains and that its effectiveness correlates with the inherent immunogenicity of the tumor. Fully human anti-CTLA4 has shown promise in clinical trials when given to patients with melanoma or ovarian cancer who had been previously immunized with GM-CSF-expressing tumor vaccines (17) or antigen-loaded DC vaccines (18) or when given concurrently with peptide vaccines (19). These studies collectively indicate the clear impact of CTLA4 blockade on tumor rejection (20). While adverse immune events have been documented in the initial clinical studies of CTLA4 blockade (21), such systemic adverse immune events have not been 


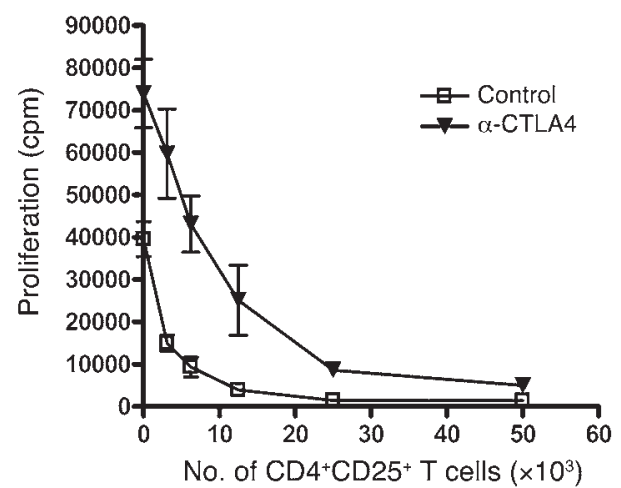

Figure 1

CTLA4 blockade enhances Teff proliferation in vitro. CD4+CD25+ Tregs and CD4+CD25- Teffs were isolated from lymph nodes and stimulated in vitro with irradiated T cell-depleted splenocytes in the presence of $10 \mu \mathrm{g} / \mathrm{ml}$ anti-CD3 in the presence or absence of 50 $\mu \mathrm{g} / \mathrm{ml}$ anti-CTLA4 monoclonal antibody. $\left[{ }^{3} \mathrm{H}\right]$ thymidine was added for the last 8 hours of a 72-hour culture. The data are representative of 3 independent experiments.

observed in murine tumor models. In the murine B16 melanoma model, vitiligo was the only documented side effect (12).

Although these effects have usually been attributed to direct interference with the function of CTLA4 on effector cells, an alternative possibility is that CTLA4 blockade may impact $\mathrm{CD} 4^{+} \mathrm{CD} 25^{+}$ Tregs. $\mathrm{CD} 4^{+} \mathrm{CD} 25^{+}$Tregs are endogenous regulators of immune responses. They constitutively express glucocorticoid-induced TNF receptor (GITR), CD103, CTLA4, and the transcription factor forkhead box protein P3 (Foxp3) and are capable of suppressing immune responses to both self and non-self $(22,23)$. Extension of work from the transplantation field, where Tregs have been shown to efficiently infiltrate skin grafts that are accepted but not those that are rejected $(24,25)$, suggests that $\mathrm{CD}^{+} \mathrm{CD} 25^{+}$Tregs might also infiltrate tumors and interact with effector $\mathrm{T}$ cells (Teffs), reducing their antitumor effect at a local level. Recent data has demonstrated the presence of infiltrating $\mathrm{CD} 4^{+} \mathrm{CD} 25^{+} \mathrm{Foxp}^{+}$ Tregs in human ovarian carcinoma (26) and in MethA murine fibrosarcoma (27). Also, in human melanoma, the level of Foxp3 message was shown to be increased in tumors from patients with metastatic disease (28). Finally, depletion of this subset prior to tumor challenge significantly increases the effectiveness of Gvax/ anti-CTLA4 combination therapy (29). Together, the data argue for the relevance of the Treg compartment in the control of the antitumor immune response.

The constitutive expression of CTLA4 on the surface of Tregs has led to the hypothesis that CTLA4 plays an essential role in their activity and that anti-CTLA4 treatment leads either to depletion of Tregs or to impairment of their suppressive activity $(30,31)$. A number of approaches have been employed to confirm the role of CTLA4 in Treg function, including CTLA4 blockade with antibodies and the use of CTLA4-knockout Tregs in both in vitro and in vivo studies (30-32). Much of the data has been interpreted as supporting an impact of anti-CTLA4 on Tregs, although it has proven difficult to exclude the possibility of a $\mathrm{T}$ cell-intrinsic mechanism of action via the direct blockade of CTLA4-mediated inhibitory signals on Teffs. Tregs from CTLA4 ${ }^{-/-}$mice appear capable of in vitro suppression (32), although it has been suggested that they do so by a different mechanism involving secretion of soluble factors such as TGF- $\beta$ rather than by direct contact-mediated mechanisms (33). A recent study of human subjects suggested that CTLA4 blockade had no impact on suppression in an in vitro assay (34). Thus, there is still controversy regarding the involvement of CTLA4 in Treg suppressive capacity, little is known about the dynamic interactions that take place between effectors and regulators during antiCTLA4-induced antitumor therapy, and even less work has been done to understand their interaction at the tumor site.

To dissect the effects of CTLA4 blockade on the balance between Teffs and Tregs during tumor rejection, we used an in vivo model of B16 melanoma and Gvax/anti-CTLA4 therapy. We clearly show the presence of $\mathrm{CD} 4^{+} \mathrm{Foxp}^{+}$Tregs within B16 melanoma tumors. The tumor-infiltrating lymphocyte (TIL) also contained $\mathrm{CD}^{+}{ }^{+} \mathrm{Foxp}^{-}{ }^{-} \mathrm{T}$ cells but relatively few $\mathrm{CD}^{+}$Teffs. In contrast, following therapeutic intervention with Gvax/anti-CTLA4, the number of CD8 ${ }^{+}$IFN- $\gamma^{+}$Teffs markedly increased within the tumor, as did the number of $\mathrm{CD}^{+}{ }^{+}$Foxp3 $3^{-} \mathrm{T}$ cells, with a resultant decrease in the percentage of infiltrating Foxp $3^{+} \mathrm{T}$ cells. Interestingly, $\mathrm{CD}^{+}{ }^{+} \mathrm{CD} 25^{+} \mathrm{Foxp}^{+}$and $\mathrm{CD} 4{ }^{+} \mathrm{CD} 25^{-}{ }^{-} \mathrm{Foxp}^{+} \mathrm{T}$ cells accumulated in the lymph nodes, most probably due to blockade of negative cellintrinsic signaling through CTLA4 and expansion in response to self antigen. In contrast, priming of tumor-reactive Teffs by Gvax induced their accumulation and activation at the tumor site in response to tumor antigen. The overt migration and enrichment of Teffs at the tumor site changed the balance between Teffs and Tregs, inducing a switch from tolerance to immunity, as supported by the tumor rejection data.

This is the first study to our knowledge to define the in vivo impact of Gvax/anti-CTLA4 therapy on the dynamic relationship between Teffs and Tregs. Moreover, we also document for the first time the differential impact of Gvax/anti-CTLA4 in the tumor and in lymph nodes. Together the data strongly suggest that the therapeutic impact of this vaccine combination resides in increases in the intratumor frequency and activity of Teffs as a consequence of priming tumor-reactive Teffs by Gvax and removal of cell-intrinsic inhibitory effects by CTLA4 blockade. Finally, the data also show that chronic exposure to anti-CTLA4 or to Gvax/ anti-CTLA4 combination therapy does not result in depletion of Tregs, nor is their regulatory activity affected. This suggests that upon cessation of therapy, the regulatory activities of these cells will be retained to help in the control of possible adverse immune events induced by CTLA4 blockade.

\section{Results}

Anti-CTLA4 increases Teff responses without blocking the capacity of Tregs to inhibit Teff proliferation in vitro. To assess the impact of anti-CTLA4 monoclonal antibody (clone 9D9) on the activity of Tregs in vitro, $\mathrm{CD} 4^{+} \mathrm{CD} 25^{-}$and $\mathrm{CD} 4^{+} \mathrm{CD} 25^{+}$Tregs were isolated from naive mice and cocultured at different ratios in the presence of anti-CD3 and irradiated APCs and in the presence or absence of $50 \mu \mathrm{g} / \mathrm{ml}$ anti-CTLA 4 or control Ig. When cultured in the absence of Tregs, $\mathrm{CD} 4^{+} \mathrm{CD} 25^{-} \mathrm{T}$ cells proliferated up to 2 -fold more when stimulated by APCs in the presence of anti-CTLA4 (Figure 1). In the presence of Tregs, the increased proliferation of the anti-CTLA4-treated group was maintained, although the effect was most noticeable at higher $\mathrm{CD} 4^{+} \mathrm{CD} 25^{-} \mathrm{T}$ cell/Treg ratios. When the same number of Tregs and $\mathrm{CD} 4^{+} \mathrm{CD} 25^{-} \mathrm{T}$ cells were cocultured, there was no significant impact of anti-CTLA4 on the suppressive capacity of Tregs. These data suggest that, in vitro, CTLA4 blockade does not directly target Treg activity but 
A

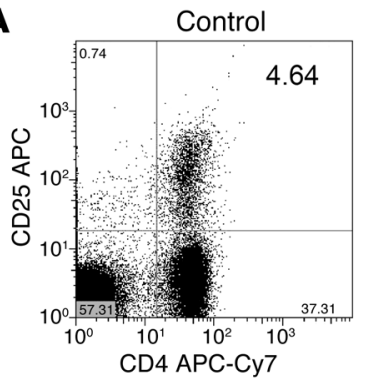

B
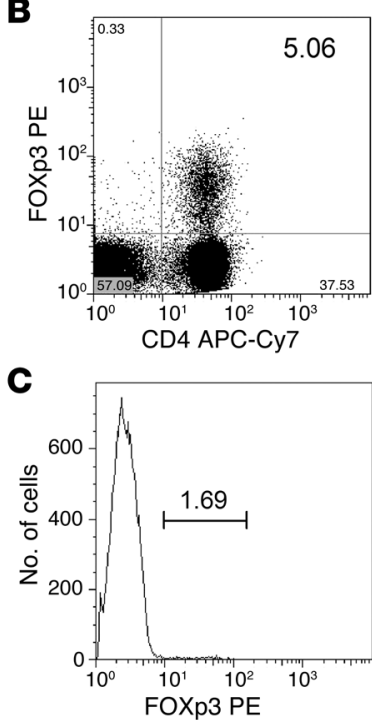

D

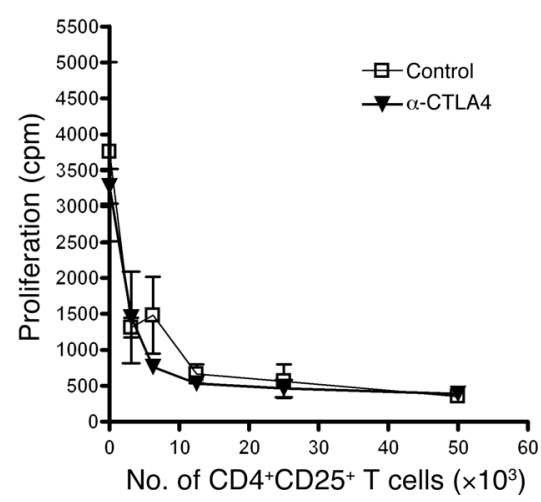

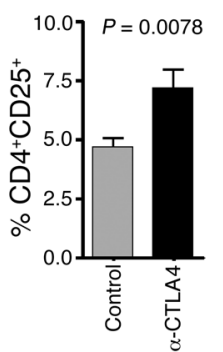
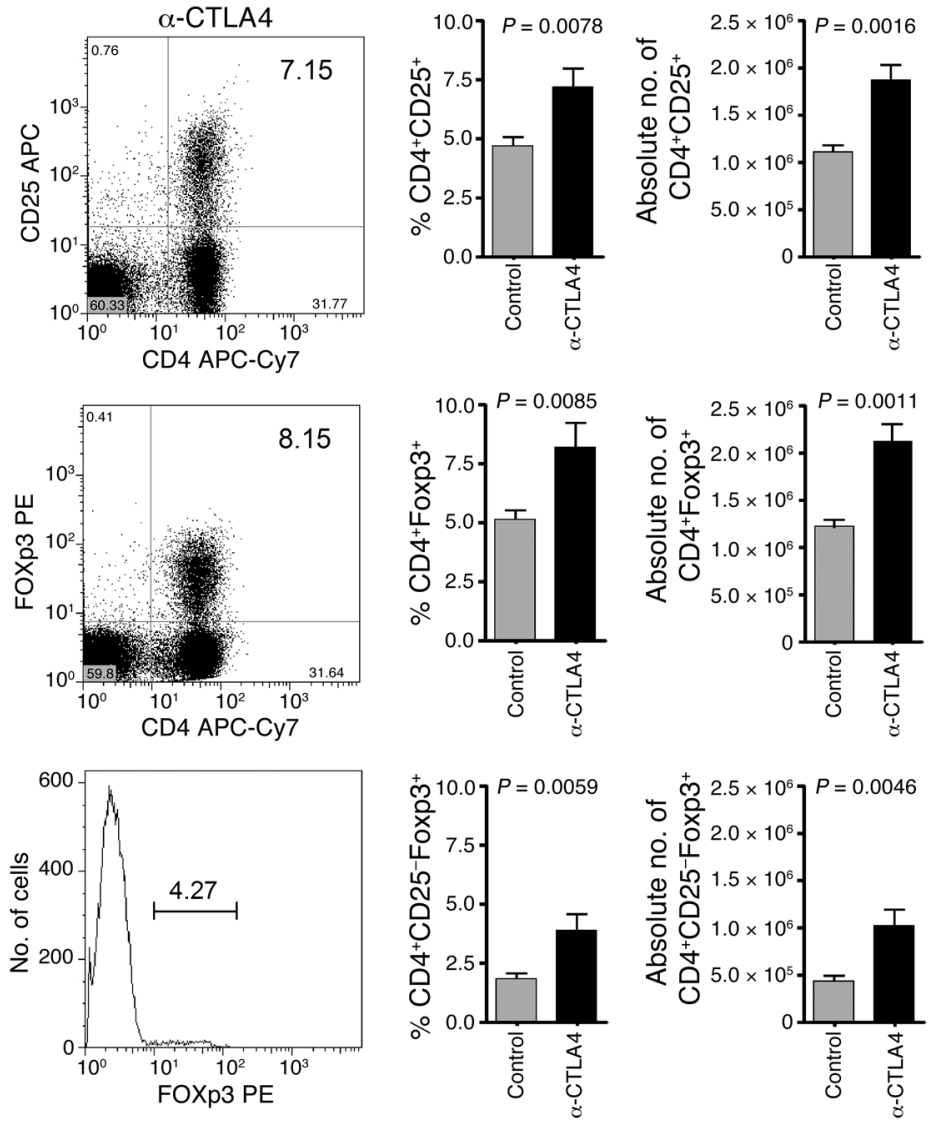

Figure 2

Chronic CTLA4 blockade increases the frequency of Tregs in the lymph nodes without permanently altering their regulatory activity. Mice were treated for a 2-week period with $100 \mu \mathrm{g}$ anti-CTLA4 or control mouse Ig every other day. Lymph nodes were harvested and stained for CD4, CD25, and Foxp3. Data are presented as cytometric dot plots and graphically as percentages and absolute numbers of $\mathrm{CD}^{+}{ }^{+} \mathrm{CD} 25^{+}$(A), CD4 ${ }^{+} \mathrm{Foxp}^{+}$ (B), and $\mathrm{CD} 4^{+} \mathrm{Foxp} 3^{+}$cells gated on the CD25- population (C). (D) $\mathrm{CD}^{+}{ }^{+} \mathrm{CD} 25^{+}$Tregs were isolated from mice treated/untreated with anti-CTLA4 for 2 weeks and tested for their ability to suppress naive $\mathrm{CD} 4{ }^{+} \mathrm{CD} 25^{-} \mathrm{T}$ cell expansion in vitro in response to irradiated T cell-depleted splenocytes and $10 \mu \mathrm{g} / \mathrm{ml}$ anti-CD3. $\left[{ }^{3} \mathrm{H}\right]$ thymidine was added for the last 8 hours of a 72-hour culture. The numbers in the upper-right corners of the dot plots represent the percentage of cells in that quadrant, and the data are representative of 3 independent experiments with 3 independently analyzed mice/group. increases $\mathrm{CD}^{+} \mathrm{CD} 25^{-} \mathrm{T}$ cell responses in a cell-intrinsic manner and renders them refractory to suppression.

Anti-CTLA4 treatment does not deplete but increases the number of Tregs in lymph nodes of mice. Since CTLA4 is constitutively expressed on Tregs, it has been suggested that the antitumor effect of CTLA4 blockade was due to depletion of Tregs and/or to interference with their activity in vivo. To test these hypotheses, we examined the impact of chronic treatment (every other day for 2 weeks) on the number and function of Tregs. Chronic anti-CTLA4 treatment did not reduce the number of Tregs in the lymph nodes whether analyzed for $\mathrm{CD}^{+} \mathrm{CD}^{2} 5^{+}$(Figure $2 \mathrm{~A}$ ) or $\mathrm{CD}^{+}{ }^{+} \mathrm{Foxp}^{+}$(Figure 2B) cells. On the contrary, chronic CTLA4 blockade consistently resulted in a significant increase in the percentages and absolute numbers of $\mathrm{CD}^{+} \mathrm{CD} 25^{+} \mathrm{T}$ cells (Figure $2 \mathrm{~A}$ ). The same was observed for $\mathrm{CD}^{+} \mathrm{CD}^{2} 5^{+} \mathrm{Foxp}^{+} \mathrm{T}$ cells (Figure $2 \mathrm{~B}$ ) and $\mathrm{CD} 4^{+} \mathrm{CD} 25^{-} \mathrm{Foxp}^{+}$ $\mathrm{T}$ cells (Figure $2 \mathrm{C}$ ). Therefore, chronic blockade of CTLA4 allows
Tregs to expand, most likely as a result of removing the restricting effects of CTLA4 on cell cycle progression.

To determine whether chronic anti-CTLA4 treatment had a negative impact on Treg activity, we tested the suppressive activity of Tregs ex vivo. To this end, $\mathrm{CD} 4^{+} \mathrm{CD} 25^{+} \mathrm{T}$ cells were isolated from mice treated with anti-CTLA4 for 2 weeks, and their ability to inhibit proliferation of $\mathrm{CD} 4^{+} \mathrm{CD} 25^{-} \mathrm{T}$ cells from untreated mice was compared with that of $\mathrm{CD} 4^{+} \mathrm{CD} 25^{+} \mathrm{T}$ cells from control mice in an in vitro suppression assay. The data presented in Figure 2D show that, on a per cell basis, there was no impact of chronic anti-CTLA4 treatment on the suppressor activity of $\mathrm{CD} 4^{+} \mathrm{CD} 25^{+} \mathrm{T}$ cells at any of the Treg/CD4 ${ }^{+} \mathrm{CD} 25^{-} \mathrm{T}$ cell ratios used. Parallel studies in our laboratory have also shown that Tregs from CTLA4 $\mathrm{Tg}$ mice that overexpress CTLA4 show no difference in their ability to suppress $\mathrm{CD} 4^{+} \mathrm{CD} 25^{-} \mathrm{T}$ cell proliferation in vitro when compared with wild-type Tregs (our unpublished 

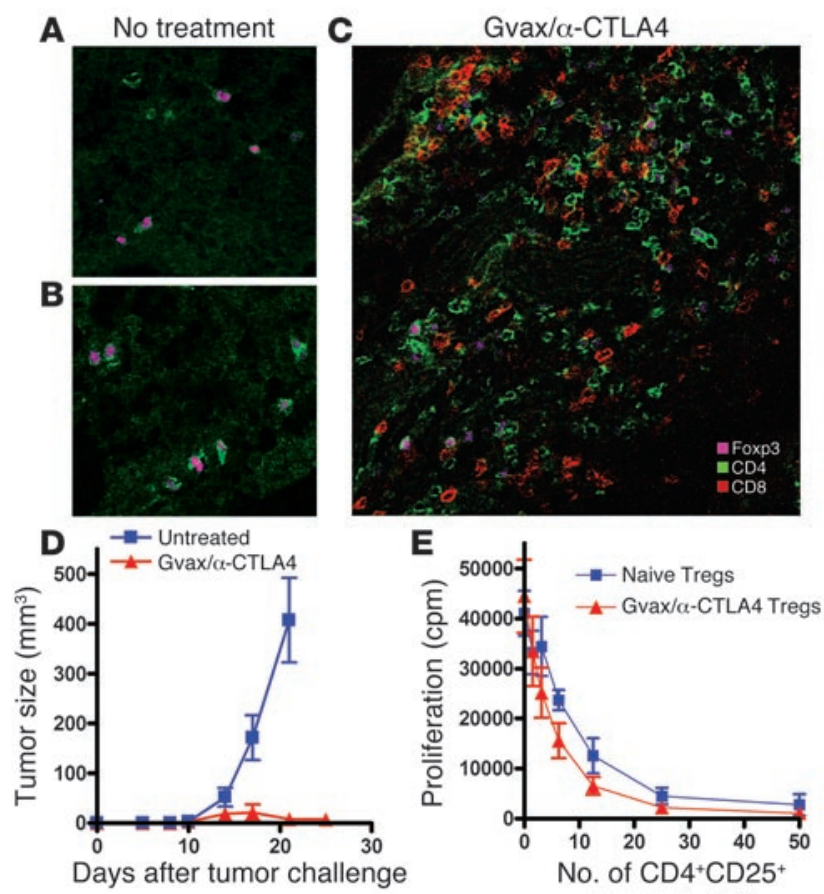

T cells $\left(\times 10^{3}\right)$

observations). Although the data do not completely exclude the possibility that anti-CTLA4 has some impact on the suppressive activity of $\mathrm{CD} 4^{+} \mathrm{CD} 25^{+}$Tregs in vivo, they strongly suggest that chronic exposure to anti-CTLA4 in vivo does not lead to depletion of $\mathrm{CD} 4^{+} \mathrm{CD} 25^{+}$Tregs nor permanently impair their suppressive function, but rather results in their expansion.

High frequencies of $C D 4^{+}$and Foxp $3^{+}$but not $C D 8^{+} T$ cells are found in B16 tumor infiltrates compared with lymph nodes during tumor progression. To evaluate the impact of Gvax and CTLA4 blockade on the Teff and Treg compartments during antitumor responses, we first studied the impact of untreated melanoma on the size of the Treg compartment within the lymph nodes and the degree of infiltration of B16 melanoma by Teffs and Tregs. For the purposes of clarity and consistency we will, henceforth, refer to all $\mathrm{CD} 4^{+} \mathrm{Foxp}^{+}$ $\mathrm{T}$ cells as Tregs and further define $\mathrm{CD} 25^{+}$and $\mathrm{CD} 25^{-}$subpopulations when necessary. Likewise we will refer to all $\mathrm{T}$ cells not within this category, whether naive or activated, as Teffs, recognizing that not all cells within this group would conventionally be considered as effector cells, and further define $\mathrm{CD}^{+}$or $\mathrm{CD}^{+}$ subsets as necessary. Mice were injected intradermally (i.d.) with the highly tumorigenic cell line B16/BL6 and sacrificed 15 days later for analysis. Confocal microscopy demonstrated the dominant intratumor infiltration to be by Tregs, with a paucity of $\mathrm{CD} 8^{+}$ Teffs (Figure 3, A and B), which correlated with fast growth and lack of tumor rejection (Figure 3D). Analysis by flow cytometry revealed an increase in the percentage of Tregs in lymph nodes upon tumor challenge (Figure 4A) and a more dramatic increase in the percentage of CD $4^{+} \mathrm{Foxp}^{3+} \mathrm{CD} 25^{-}$Tregs (Figure 4B). Figure 4C shows the distribution of $\mathrm{CD}^{+}, \mathrm{CD}^{+}$, and Foxp $3^{+} \mathrm{T}$ cells in lymph nodes from tumor-bearing mice, while Figure $4 \mathrm{D}$ shows their frequency in TILs 15 days after tumor challenge. In comparison with the findings in lymph nodes, $\mathrm{CD}^{+} \mathrm{Teffs}$ were underrepresented in the tumor when compared with the percentage of Tregs and $\mathrm{CD} 4^{+}$ Teffs. Most importantly, we observed that the ratio of $\mathrm{CD} 4^{+}$Teffs

\section{Figure 3}

Gvax/anti-CTLA4 combination therapy induces tumor infiltration and rejection without permanently impairing Treg function. Immunofluorescence microscopy of 8- $\mu$ m-thick sections from 15-dayold tumors from untreated mice (A and $\mathbf{B}$ ) and mice treated with Gvax/ anti-CTLA4 (C). Tumor sections were fixed in acetone and stained with anti-CD4-FITC (green), anti-CD8-PE (red), and anti-Foxp3 $3^{+}$APC (pink). Samples were analyzed with a Leica Microsystems inverted confocal microscope with $a \times 20$ water immersion objective. The data are representative of 3 independent experiments with 3 mice/group. (D) In parallel experiments, mice ( $n=10$ mice per group) were monitored for tumor growth and rejection upon treatment with Gvax/anti-CTLA4 combination therapy. (E) CD4+CD25+ Tregs from tumor-draining lymph nodes of Gvax/anti-CTLA4-treated mice or naive mice were tested for their ability to suppress CD4+CD25- T cell proliferation in vitro.

to Tregs was lower in tumors than in lymph nodes $(P=0.0008)$ and, more strikingly, that intratumor $\mathrm{CD}^{+}$Teffs were present, on average, at about a 1:1 ratio when compared with Tregs (Figure 4E). Hence tumor-bearing mice show not only a significantly higher frequency of Tregs in lymph nodes but also significant infiltration of tumors by $\mathrm{CD} 4^{+}$Teffs and Tregs, with relatively few intratumoral $\mathrm{CD}^{+}$Teffs.

Anti-CTLA4/Gvax antitumor therapy increases $\mathrm{CD} 4^{+}$Teff/Treg ratios at the tumor site. While a few studies have documented the capacity of Foxp $3^{+} \mathrm{T}$ cells to infiltrate tumors $(26,27)$, little is known with regard to the cellular dynamics in the tumor versus the lymph nodes, and even less is known with regard to the changes that take place upon antitumor therapeutic intervention. Hence, we studied the impact of Gvax/anti-CTLA4 antitumor therapy on the Teff and Treg compartments in the tumor as well as in the lymph nodes using B16/BL6 melanoma in Matrigel. Briefly, mice were injected with B16/BL6 cells i.d. in Matrigel and were treated or not with B16-Gvax cells and anti-CTLA4. Fifteen days after tumor challenge, lymph nodes and TILs were analyzed by flow cytometry.

As shown in Figure 5A, the percentage of Tregs increased in lymph nodes upon Gvax/anti-CTLA4 treatment, resulting in a decrease in the ratio of Teffs to Tregs in lymph nodes upon therapy (Figure 5B). This observation correlates with data presented in Figure 2, which show that the percentage of Tregs was increased upon chronic anti-CTLA4 treatment. The same pattern was observed whether analysis was restricted to the tumor-draining lymph nodes or included distal nodes (Supplemental Figure $2 \mathrm{~A}$; supplemental material available online with this article; doi:10.1172/JCI27745DS1). Interestingly, a profoundly different effect was observed in the tumor. As shown in Figure 5C, the percentage of Tregs in TILs was reduced upon Gvax/anti-CTLA4 treatment, while the percentage of $\mathrm{CD} 4^{+}$Teffs was increased. Gvax/anti-CTLA4 therapy caused a significant increase in the ratio of $\mathrm{CD}^{+}$Teffs to Tregs $(P=0.0151)$ (Figure $5 \mathrm{D}$ ), which correlated with tumor infiltration (Figure 3C) and tumor rejection (Figure 3D). In addition, Tregs isolated from tumor-draining lymph nodes of Gvax/anti-CTLA4-treated mice showed the same suppressive capacity as Tregs isolated from naive mice (Figure 3E), suggesting that the impact of the therapy on tumor infiltration and rejection is not related to a lasting effect of the therapy upon the Treg compartment. Therefore, in lymph nodes, Gvax/anti-CTLA4 therapy induces a decrease in the ratio of $\mathrm{CD} 4^{+}$Teffs to Tregs, probably as a consequence of increased proliferation of Tregs chronically stimulated by self antigen. The reverse takes place at the tumor site, 

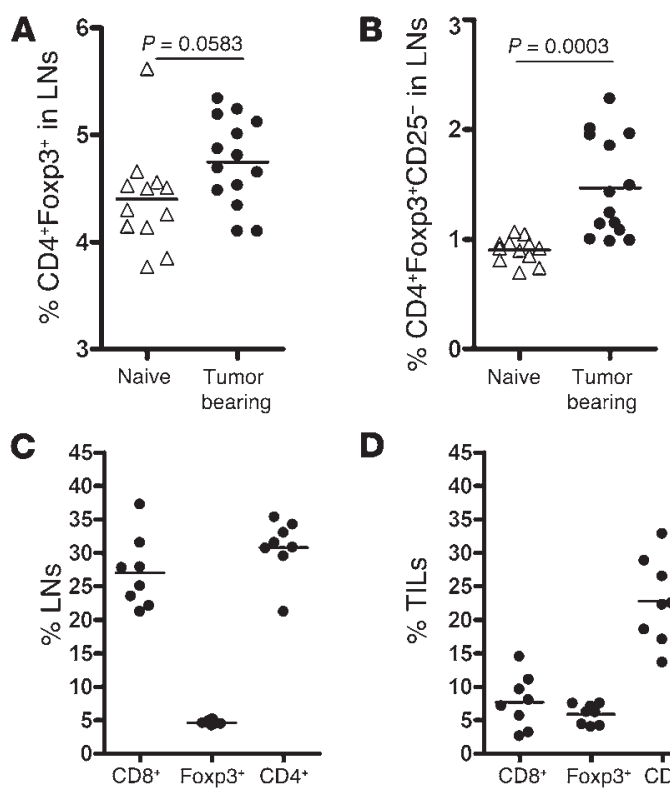

D
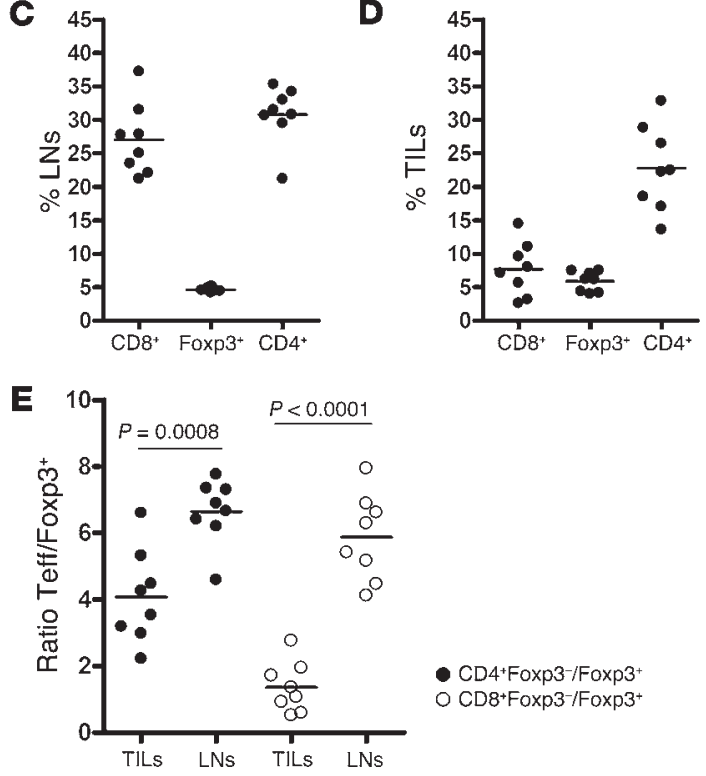

where the ratio of $\mathrm{CD} 4^{+}$Teffs to Tregs is dramatically increased $(P=0.0151)$, probably as a result of increased expansion and/or recruitment of tumor-specific $\mathrm{CD}^{+}{ }^{+}$Teffs allowed by removal of CTLA4-mediated inhibition, and without lasting impairment of Treg function.

Anti-CTLA4/Gvax antitumor therapy markedly increases intratumor $C D 8^{+} T e f f / T r e g$ ratios. It has been demonstrated that the Gvax/anti-CTLA4-induced antitumor effector function is predominantly mediated by $\mathrm{CD}^{+} \mathrm{T}$ cells (12). We therefore studied the impact of this treatment on the function of intratumor $\mathrm{CD}^{+} \mathrm{T}$ cells and their interaction with Tregs. As observed for $\mathrm{CD}^{+} \mathrm{T}$ cells, the ratio of $\mathrm{CD}^{+}$ Teffs to Tregs was also significantly decreased in the lymph nodes upon Gvax/anti-CTLA4 treatment

\section{Figure 5}

Gvax/anti-CTLA4 treatment increases the ratio of effector $\mathrm{CD}^{+} \mathrm{T}$ cells to Tregs in the tumor but not in the lymph nodes. Fifteen days after tumor challenge, lymph nodes (A and $\mathbf{B}$ ) and tumors ( $\mathbf{C}$ and $\mathbf{D}$ ) from untreated or Gvax/ anti-CTLA4-treated mice were analyzed by flow cytometry for their content of CD4+ and Foxp3 ${ }^{+}$T cells. A and $\mathbf{C}$ show representative dot plots for untreated and treated mice, while $\mathbf{B}$ and $\mathbf{D}$ show the ratio of $\mathrm{CD}^{+}{ }^{+}$Foxp3 $3^{-}$to CD4 ${ }^{+}$Foxp $3^{+}$cells in 1 of 3 representative experiments with 3 independently analyzed mice/group.

\section{Figure 4}

B16 melanoma TILs contain a high frequency of $\mathrm{CD}^{+}$and $\mathrm{Foxp}^{+}$ but few $\mathrm{CD}^{+} \mathrm{T}$ cells. The percentage of $\mathrm{CD} 4{ }^{+} \mathrm{Foxp} 3^{+} \mathrm{T}$ cells $(\mathbf{A})$ and CD4+Foxp3+CD25-T cells (B) was analyzed by flow cytometry of lymph nodes from naive and tumor-bearing mice 15 days after tumor challenge. In a parallel set of studies, lymph nodes (C) and tumors (D) from tumor-bearing mice were analyzed at day 15 for their content of CD8 ${ }^{+}$, Foxp3 $^{+}$, and $\mathrm{CD}^{+}{ }^{+} \mathrm{T}$ cells. (E) The ratio of CD4+Foxp3- to Foxp3 ${ }^{+} \mathrm{T}$ cells (filled circles) and CD8 ${ }^{+}$Foxp3- to Foxp3 ${ }^{+}$T cells (open circles) was calculated and compared in tumors and lymph nodes from tumorbearing mice 15 days after tumor challenge. The data represent cumulative results from 3 independent experiments with 3-5 mice/group.

$(P=0.0239)$ (Figure 6, A and B). Again, the same pattern of changes was revealed by analysis of tumor-draining versus total nodes (Supplemental Figure 2B). Analysis of TILs showed 7.11\% CD8 ${ }^{+} \mathrm{T}$ cells and $4.15 \%$ Foxp $^{+}$cells (Figure 6C). This changed dramatically upon Gvax/anti-CTLA4 treatment, when the percentage of CD8 ${ }^{+}$ $\mathrm{T}$ cells reached $33.11 \%$ of the TILs and the ratio of $\mathrm{CD}^{+}$Teffs to Tregs increased from an average of 1.8 to $12.9(P=0.0345)$ (Figure $6 \mathrm{D})$. The result of this was efficient tumor rejection (Figure 3D). When analyzing the independent contributions of anti-CTLA4 and Gvax to antitumor responses, we observed that only combination therapy was fully effective at inducing tumor rejection $(P=0.0007$ compared with untreated mice), while Gvax alone induced only a delay in tumor growth $(P=0.4737$ compared with untreated mice) and anti-CTLA4 monotherapy had no effect (Supplemental Figure 1A). These observations correlate with the findings that the most dramatic change in the ratio of CD8 ${ }^{+}$Teffs to Tregs was observed during Gvax/anti-CTLA4 combination therapy; that Gvax monotherapy only induced a small increase in this ratio; and that anti-CTLA4 monotherapy did not induce an increase in the intratumor $\mathrm{CD}^{+}$Teff/Treg ratio (Supplemental Figure 1B). Confocal microscopy confirmed that tumors from untreated mice showed dominant infiltration by Tregs and mini-
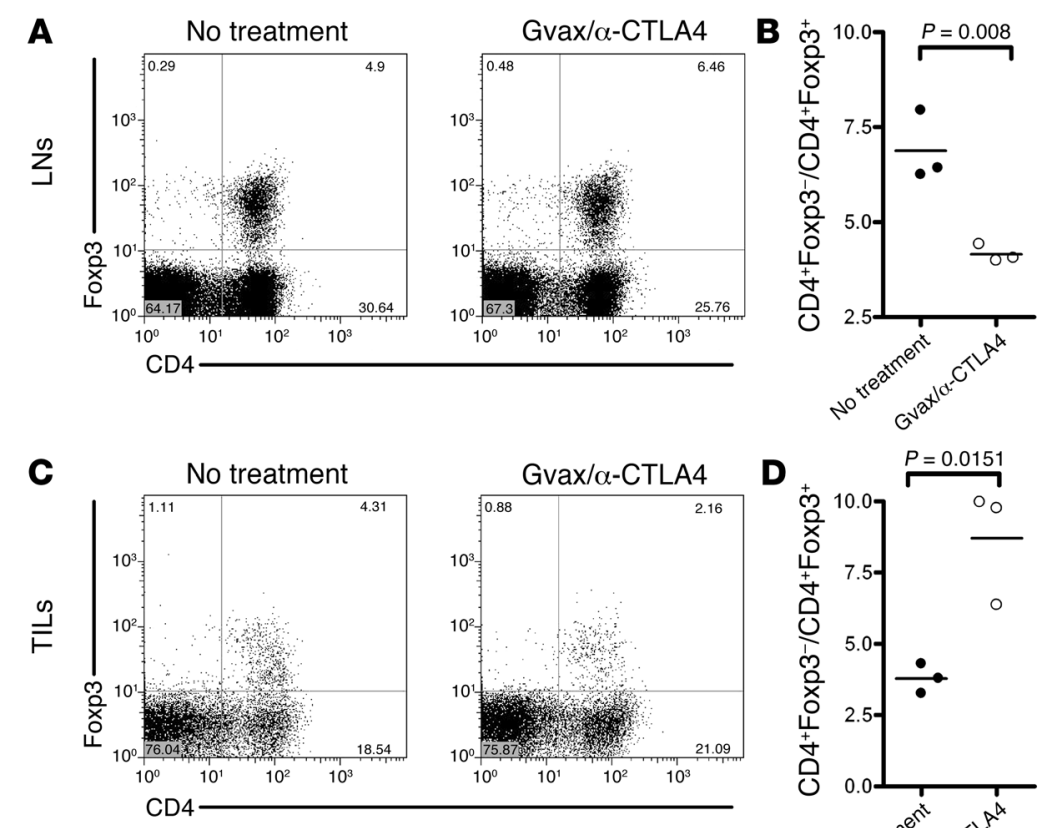

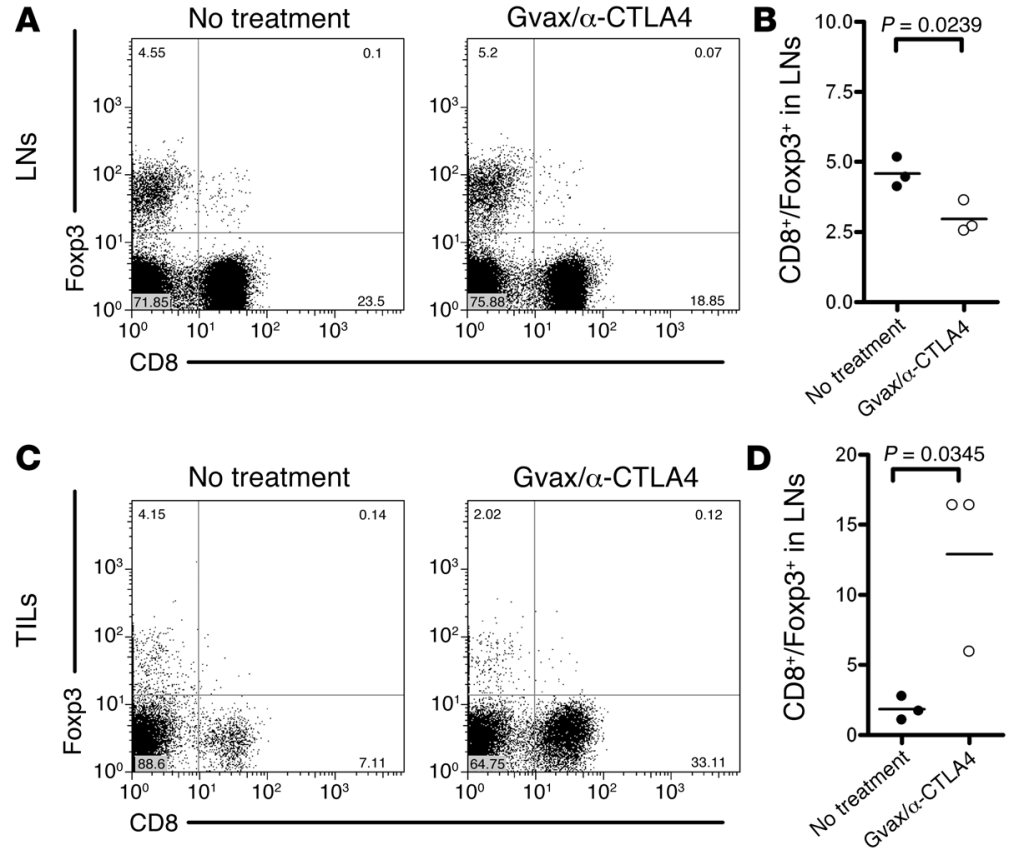

\section{Figure 6}

Gvax/ $\alpha$ CTLA4 treatment increase the ratio of effector CD8 ${ }^{+} \mathrm{T}$ cells to Tregs in the tumor but not in the lymph nodes. Fifteen days after tumor challenge, lymph nodes (A and $\mathbf{B}$ ) and tumors ( $\mathbf{C}$ and $\mathbf{D})$ from untreated or Gvax/ anti-CTLA4-treated mice where analyzed by flow cytometry for their content of CD8 $8^{+}$and Foxp3 ${ }^{+} \mathrm{T}$ cells. A and C show representative dot plots for untreated and treated mice, while $\mathbf{B}$ and $\mathbf{D}$ show the ratio of $\mathrm{CD}^{+}$to $\mathrm{Foxp}^{+}$ cells in 1 of 3 representative experiments with 3 independently analyzed mice/group. mal CD8 ${ }^{+} \mathrm{T}$ cell infiltration (Figure 3, A and B), while tumors from mice treated with combination immunotherapy showed increased infiltration by CD8- and CD4-single-positive cells. Tregs were still observed but at an obvious numerical disadvantage when compared with CD4- and CD8-single-positive cells (Figure 3C). These results suggest that Gvax exerts its effect by efficiently priming tumor-reactive $\mathrm{CD}^{+}$Teffs, but subsequent expansion and differentiation as a result of CTLA4 blockade is necessary in order to induce efficient antitumor responses.

Anti-CTLA4/Gvax increases the absolute number and activity of tumor-infiltrating $C D 8^{+} T$ cells. To assess the quantity and quality of $\mathrm{CD}^{+} \mathrm{T}$ cell infiltrates following Gvax/anti-CTLA4 treatment, we determined the absolute number of TILs and analyzed the expression of the activation marker CD25 and the effector cytokine IFN- $\gamma$. Upon therapeutic intervention, the absolute number of $\mathrm{CD}^{+}$cells/gram tumor was increased by approximately 10-fold (Figure 7A). These cells upregulated CD25 (Figure $7 \mathrm{~B}$ ) and were capable of producing higher amounts of IFN- $\gamma$ in response to restimulation with the tumor-derived peptide TRP2 (Figure 7C), as well as with PMA/ionomycin restimulation (Figure 7D). When analyzing the independent contributions of Gvax and anti-CTLA4 to CD8 ${ }^{+} \mathrm{T}$ cell infiltration and differentiation, we observed that Gvax alone also induced an increase in $\mathrm{CD}^{+} \mathrm{T}$ cell infiltration, while CTLA4 blockade alone did not induce a significant change (Supplemental Figure 1C). Also, Gvax alone or in combination with anti-CTLA4 induced IFN- $\gamma$ production by the infiltrating $\mathrm{CD}^{+} \mathrm{T}$ cells (Supplemental Figure 1D). The number of $\mathrm{CD} 4^{+}$cells/gram of tumor also increased, but only in the Gvax/anti-CTLA4-treated group (Supplemental Figure 1E), while the number of Tregs/gram of tumor remained the same in all treated groups (data not shown).

Thus, CTLA4 blockade and Gvax combination therapy show no direct impact on tumor infiltration by Tregs but significantly increase the absolute number of infiltrating $\mathrm{CD} 8^{+} \mathrm{Teffs}$; the ratio of $\mathrm{CD}^{+}$Teffs to Tregs; and the differentiation of $\mathrm{CD}^{+}$naive $\mathrm{T}$ cells into $\mathrm{CD}^{+} \mathrm{CD}^{+} 5^{+}$and $\mathrm{CD} 8{ }^{+} \mathrm{IFN}-\gamma^{+} \mathrm{T}$ cells via a combination of efficient priming and removal of the restraining effects of CTLA4 on $\mathrm{T}$ cell cycle progression.

Since most clinical studies in humans rely on blood as the analyte for laboratory monitoring of antitumor responses, we performed a series of assays on murine blood samples. These included assays for $\mathrm{CD}^{+}{ }^{+} \mathrm{CD} 25^{+} \mathrm{Foxp}^{+} \mathrm{T}$ cells, total $\mathrm{CD} 8^{+} \mathrm{T}$ cells, and tumor-specific $\mathrm{T}$ cells by IFN- $\gamma$ production in response to a number of restimulation protocols with irradiated B16 tumor, TRP2 peptide-loaded B16 tumor, or anti-CD3 monoclonal antibody. We were unable to detect IFN- $\gamma$ production directly in response to tumor restimulation at the time point analyzed (day 15). Overnight stimulation with anti-CD3 allowed us to detect low percentages of $\mathrm{CD}^{+}$IFN- $\gamma$-producing T cells. When we analyzed the percentage of $\mathrm{CD}^{+} \mathrm{T}$ cells or the ratio of $\mathrm{CD}^{+} \mathrm{T}$ cells to Tregs, we found a small increase that correlated with therapeutic intervention (Supplementary Figure 3). These data suggest that the changes observed in the tumor and lymph nodes are not clearly detectable in blood samples and that blood might not be the most informative analyte for clinical studies.

$C D 4^{+} C D 25^{+}$Tregs suppress nonspecific and tumor-specific $C D 8^{+} T$ cell responses. To further determine the direct impact of Tregs on CD8 $\mathrm{T}$ cell activity, we assessed the capacity of Tregs to control CD8 responses both in vitro and in vivo. Briefly, CD $4^{+} \mathrm{CD} 25^{+}$Tregs isolated from naive mice or Gvax/anti-CTLA4-treated mice were coincubated with 100,000 naive $\mathrm{CD}^{+} \mathrm{T}$ cells in the presence of irradiated $\mathrm{T}$ cell-depleted splenocytes and anti-CD3. $\mathrm{CD}^{+} \mathrm{T}$ cell proliferation was reduced by almost $50 \%$ in the presence of small numbers of Tregs from naive or Gvax/anti-CTLA4-treated mice (Figure $8 \mathrm{~A}$ ), suggesting that Tregs can inhibit $\mathrm{CD}^{+} \mathrm{T}$ cell proliferation and that this Treg activity is not impaired after Gvax/ anti-CTLA4 therapy (as also observed for CD4 T cell proliferative responses; Figure $3 \mathrm{E}$ ). To test whether $\mathrm{CD} 4^{+} \mathrm{CD} 25^{+}$Tregs are capable of suppressing tumor-specific $\mathrm{CD}^{+} \mathrm{T}$ cell responses, we isolated $\mathrm{CD}^{+}$TILs from mice challenged with B16/BL6 tumors and treated with Gvax on days 3, 6, and 9. When CD8 ${ }^{+}$TILs were cocultured with DCs and irradiated B16/BL6 cells, 36.7\% of the 


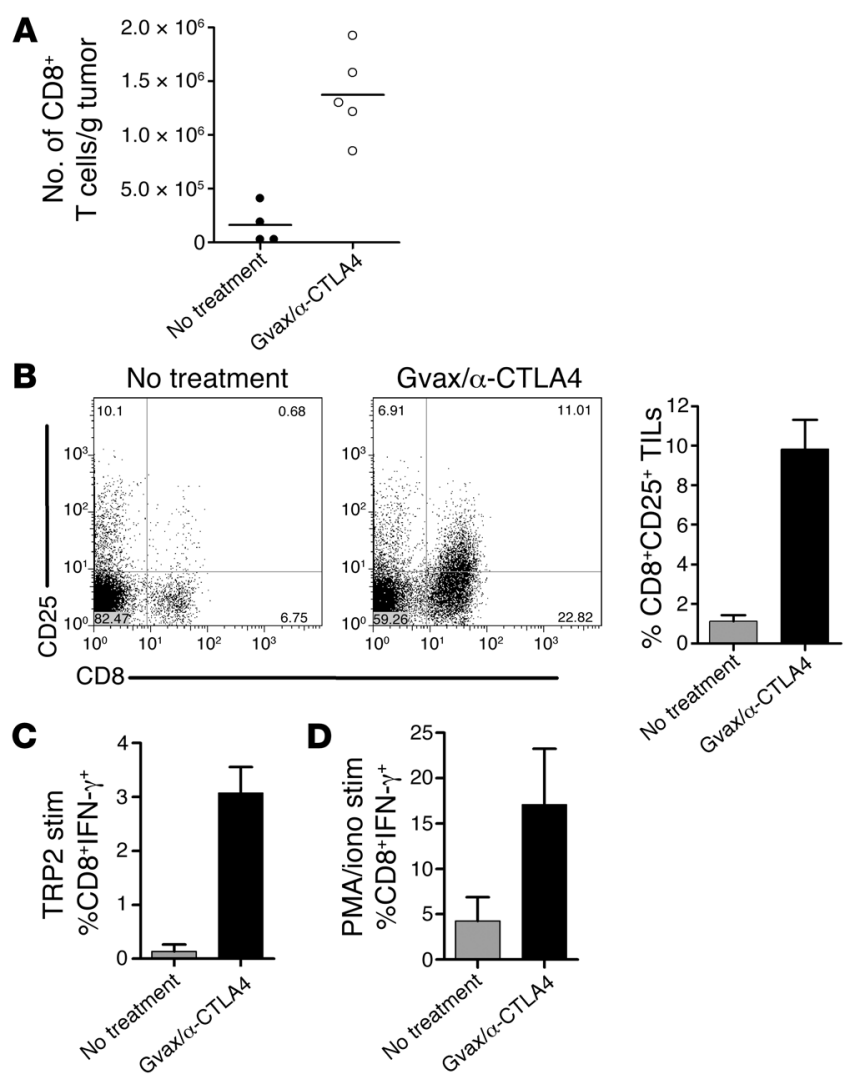

$\mathrm{CD}^{+} \mathrm{T}$ cells produced high levels of IFN- $\gamma$ (MFI, 951) compared with control groups with unloaded DCs $(8.9 \%)$ or DCs coincubated with TRAMP, an alternate nonmelanoma control tumor (11\%). The addition of $\mathrm{CD} 4{ }^{+} \mathrm{CD} 25^{+}$Tregs resulted in a reduction in both the percentage of $\mathrm{CD}^{+} \mathrm{T}$ cells producing IFN- $\gamma$ (15.3\%) and the amount of IFN- $\gamma$ produced as assessed by a reduction in the MFI (to 565; Figure 8B). These data show the negative impact that Tregs can exert on tumor-specific $\mathrm{CD}^{+} \mathrm{T}$ cell responses.

To further demonstrate the impact of $\mathrm{CD} 4{ }^{+} \mathrm{CD} 25^{+}$Tregs on $\mathrm{CD}^{+} \mathrm{T}$ cell infiltration and tumor rejection in vivo, we depleted $\mathrm{CD} 25^{+} \mathrm{T}$ cells with a monoclonal antibody (clone PC61) prior to tumor challenge and Gvax therapy. Previous studies have demonstrated that anti-CD25 synergizes with anti-CTLA4 in the same model of B16 melanoma, producing a significantly greater antitumor effect when administered with Gvax (100\% tumor protection) than when either agent was given independently along with Gvax therapy (50\% protection in both cases) (29). Although interpretation of some functional outcomes of CD25 depletion may be complicated by concurrent depletion of activated Teffs, these data are consistent with an independent mechanisms of action of CD25 depletion versus CTLA4 blockade and support an effect of antiCTLA4 predominantly on the effector compartment. In the current experiment, we aimed only to demonstrate whether removal of the $\mathrm{CD} 25^{+}$regulatory cell compartment resulted in effects on the CD8 compartment within the tumor. The results demonstrate a significant increase in $\mathrm{CD}^{+} \mathrm{T}$ cell infiltration of the tumor at day 15 (Figure $8 \mathrm{C}$ ) that strongly correlated with tumor rejection (Figure 8D). Overall, the data suggest that $\mathrm{CD} 4^{+} \mathrm{CD} 25^{+}$Tregs are capable of suppressing tumor-specific $\mathrm{CD}^{+} \mathrm{T}$ cell responses both in vitro and in vivo.

\section{Figure 7}

Gvax/anti-CTLA4 treatment increases the frequency and number of functional CD8 ${ }^{+} \mathrm{T}$ cells in tumors. C57BL/6 mice were challenged with B16/BL6 tumor at day 0 and treated/not treated with Gvax/anti-CTLA4 at days 3, 6, and 9. (A) Fifteen days after tumor challenge, tumors were removed, weighed, and analyzed by flow cytometry for the number of infiltrating $\mathrm{CD}^{+} \mathrm{T}$ cells. The remaining cells were analyzed for the expression of CD25 (B) or restimulated in vitro with either TRP2 peptide (C, TRP2 stim) or PMA/ionomycin (D, PMA/iono stim) and analyzed for intracellular expression of IFN- $\gamma$ as specified in Methods. The data are representative of 3 independent experiments with 3-5 independently analyzed mice/group.

\section{Discussion}

We have used the poorly immunogenic B16/BL6 mouse melanoma as an in vivo model to analyze the impact of anti-CTLA4 and Gvax on the temporal dynamics of Teffs and Tregs in both the lymph nodes and the tumor. The results presented here have significant relevance for our understanding of the mechanisms by which CTLA4 blockade regulates responses of Teffs and Tregs and provide insight into the way in which this immunotherapeutic approach affects the interplay of these $T$ cells in enhancing tumor rejection. First, we show that anti-CTLA4 enhances CD4 ${ }^{+}$ Teff proliferation even in the absence of Tregs in vitro. Second, anti-CTLA4 has no apparent negative impact on the suppressive capacity of Tregs in vitro, nor does chronic exposure to antiCTLA4 or Gvax/anti-CTLA4 in vivo impair Treg activity ex vivo. Third, anti-CTLA4 does not induce depletion of the CD $4^{+} \mathrm{CD} 25^{+}$ Treg population in treated mice, but, in contrast, it provokes their expansion in the lymph nodes. As a consequence of this expansion of Tregs, the ratio of both $\mathrm{CD}^{+}$Teffs and $\mathrm{CD}^{+}$Teffs to Tregs actually decreases in the lymph node. Finally, while the TILs of untreated tumors comprise mostly $\mathrm{CD} 4^{+}$Teffs and Tregs but few $\mathrm{CD}^{+}$Teffs, therapy results in a marked increase in CD4 ${ }^{+}$Teffs and, even more strikingly, $\mathrm{CD}^{+}{ }^{+}$Teffs.

It has previously been suggested that CTLA4 blockade interferes with Treg activity in vivo and in vitro $(30,31)$. In a model of induced colitis in which $\mathrm{CD} 4{ }^{+} \mathrm{CD} 25^{+}$Tregs protected against $\mathrm{CD}^{+} \mathrm{CD} 25^{-} \mathrm{T}$ cell-induced pathology, the addition of antiCTLA4 antibodies abrogated protection (30). While these results were interpreted as indicating that the mode of action of CTLA4 blockade was solely at the level of the Tregs, a cell-autonomous impact on the Teff compartment could not be excluded. Assessment of Treg activity in vitro had also suggested that CTLA4 blockade could inhibit their function in some but not all studies $(31,34)$. We found a direct impact of anti-CTLA4 on CD4 ${ }^{+}$Teffs in the absence of Tregs, suggesting that blockade of Treg function is not the only mechanism by which anti-CTLA4 can enhance Teff proliferation. Moreover, one would expect that if CTLA4 is essential for Treg function, blockade of CTLA4 should abolish suppression at any ratio of Teffs to Tregs. However, we observed that anti-CTLA4, at a 1:1 ratio of Teffs to Tregs, has a minimal impact on $\mathrm{T}$ cell proliferation. It was also reported that in the absence of Tregs (CD25-depleted mice), the addition of low doses of anti-CTLA4 could effectively induce colitis, suggesting that the effect of the antibody resides at the level of the Teff compartment (31). Also, the synergy between CTLA4 blockade and CD $4^{+} \mathrm{CD} 25^{+}$ Treg depletion previously demonstrated in this same B16/BL6 tumor model suggests that CTLA4 blockade exerts its effect by a mechanism other than the targeting of Treg activities (29). Our 


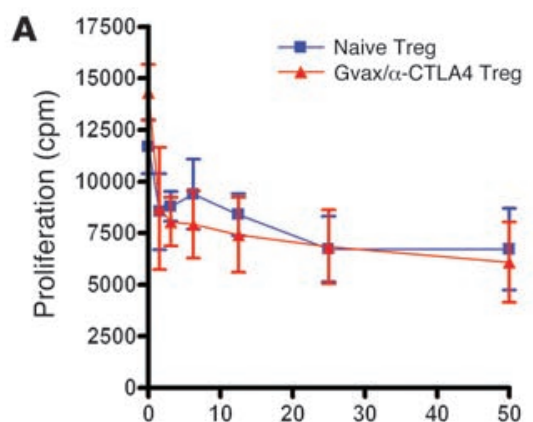

No. of $\mathrm{CD} 4{ }^{+} \mathrm{CD} 25^{+} \mathrm{T}$ cells $\left(\times 10^{3}\right)$

B

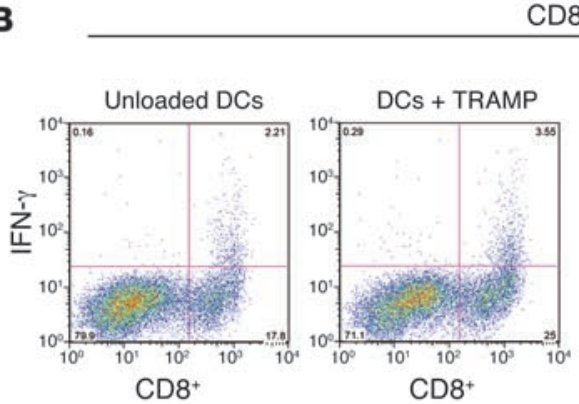

CD8+ TILs +

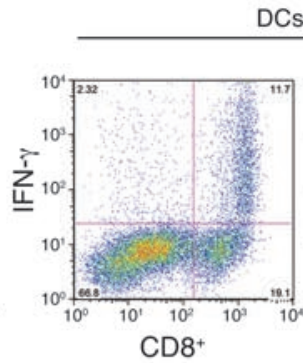

$\mathrm{DCs}+\mathrm{B} 16$
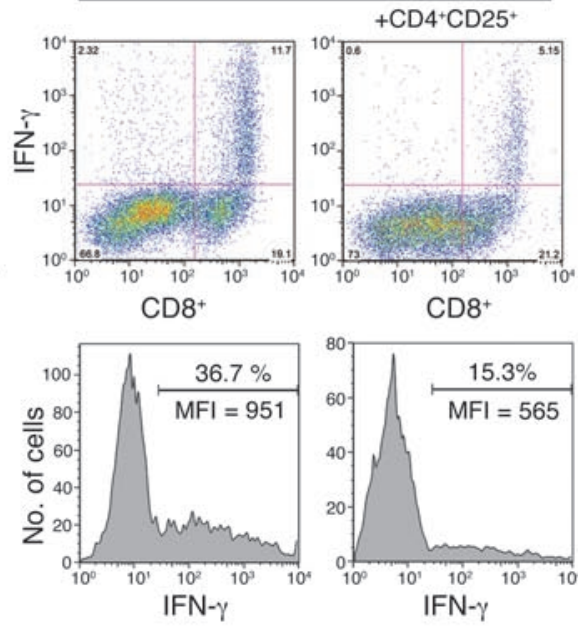

$\mathrm{CD}^{+}$

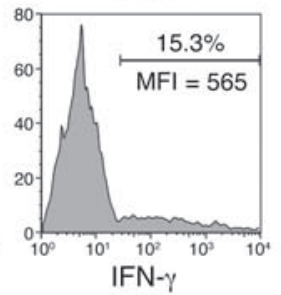

\section{Figure 8}

CD4+CD25+ Tregs suppress nonspecific and tumor-specific CD8 ${ }^{+} \mathrm{T}$ cell responses. (A) $\mathrm{CD} 4+\mathrm{CD} 25^{+}$Tregs were isolated from Gvax/anti-CTLA4-treated or naive C57BL/6 mice and tested for their ability to suppress proliferation of $\mathrm{CD}^{+} \mathrm{T}$ cells isolated from naive mice.

(B) $\mathrm{CD}^{+}{ }^{+}$TILs were isolated from B16/ BL6 tumors and restimulated overnight with DCs, DCs with irradiated TRAMP tumor, or DCs with irradiated B16/BL6 tumors in the presence or absence of naive CD4 ${ }^{+}$CD $25^{+}$Tregs. Monensin was added for the last 4 hours of culture, and production of IFN- $\gamma$ was determined by flow cytometry. In an additional set of experiments $\mathrm{C} 57 \mathrm{BL} / 6$ mice were challenged with B16/BL6 and left untreated or treated with Gvax at days 3, 6, and 9 or anti-CD25 at day -4 and Gvax at days 3,6 , and 9. (C) Mice were sacrificed 15 days after tumor challenge, and tumors were removed, weighed, and analyzed by flow cytometry to determine the number of infiltrating $\mathrm{CD}^{+} \mathrm{T}$ cells. $P=0.0029$, Gvax vs. Gvax/anti-CD25. (D) In parallel experiments, tumor growth and rejection were followed over time in the different groups. The numbers of mice/group rejecting tumors were: untreated, 0/5; Gvax, 2/10 ( $P=0.5238)$; Gvax/anti-CD25, 7/10 ( $P=0.0256)$. Data are representative of 2 independent experiments.

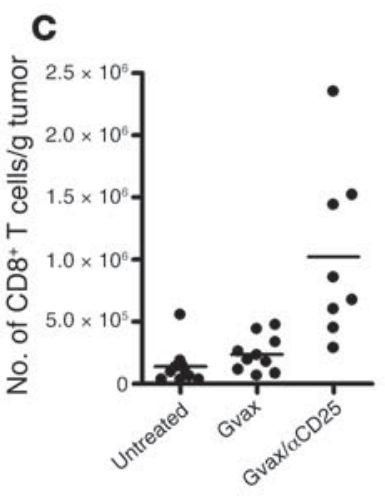

D

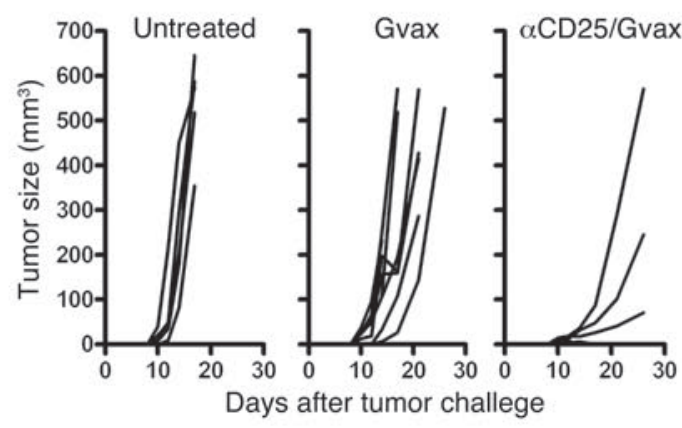

demonstration that chronic exposure (2 weeks) to anti-CTLA4 in vivo has no effect on the subsequent suppressive capacity of Tregs ex vivo is in keeping with previous results showing that chronic exposure to anti-CTLA4 during culture of Tregs in vitro did not affect their regulatory activity (35). We believe that although the data presented do not fully eliminate the possibility that CTLA4 blockade has some impact on Treg activity in vivo, they strongly suggests that CTLA4 blockade has a major impact on Teff activity and does not permanently interfere with Treg activity.

Our study also indicates that chronic anti-CTLA4 treatment does not cause depletion of Tregs. Depletion of Tregs has been suggested as an alternative mechanism of action of anti-CTLA4 therapy in vivo, in part based on the fact that Tregs constitutively express CTLA4. Preclinical studies, however, showed no evidence of depletion of $\mathrm{CD} 4^{+} \mathrm{CD} 25^{+} \mathrm{T}$ cells in the blood of cynomolgus macaques (36). In some clinical trials of anti-CTLA4 therapy, a small reduction in the percentage of $\mathrm{CD} 4^{+} \mathrm{CD} 25^{+} \mathrm{T}$ cells in the blood was reported, although absolute numbers were not documented (37). In contrast, a recent evaluation of human subjects participating in clinical studies of CTLA4 blockade suggested that Foxp $3^{+}$gene expression assessed by RT-PCR was increased in some individuals following therapy, and this was interpreted as indicating a possible expansion of the regulatory compartment (34). However, the numbers of cells expressing Foxp $3^{+}$protein were not enumerated, and in addition there appeared to be no correlation of these changes with clinical outcomes, leaving the issue of the relevance of the findings inconclusive. We show here that chronic CTLA4 blockade not only does not deplete but actu- 
ally results in increased numbers of $\mathrm{CD} 25^{+}$and $\mathrm{CD} 25^{-}$Tregs in the lymph nodes of mice. It seems likely that this is due to the same mechanisms by which CTLA4 blockade enhances proliferation of activated Teffs, such as removal of constraints on IL-2 production and cyclins that limit proliferation. The fact that antiCTLA4 does not deplete the normal repertoire of Tregs and does not permanently impair their activity may be extremely relevant in a clinical setting, as these cells may contribute to the reversal of adverse immune events revealed in clinical studies following cessation of antibody therapy $(20,37)$.

Our data demonstrate that tumor engraftment in itself induces the accumulation of both $\mathrm{CD} 4^{+} \mathrm{CD} 25^{+} \mathrm{Foxp}^{+}$and $\mathrm{CD} 4^{+} \mathrm{CD} 25^{-}$ $\mathrm{Foxp}^{+} \mathrm{T}$ cells in lymph nodes. This result is consistent with recent data suggesting that tumors are capable of inducing TGF- $\beta$ secreting DCs that selectively promote the proliferation of Tregs in lymph nodes (38). Expansion of CD $4^{+} \mathrm{CD} 25^{-}$Foxp3 $3^{+}$Tregs suggests that therapeutic interventions aimed solely at depletion of $\mathrm{CD}_{25} 5^{+} \mathrm{T}$ cells might not be sufficient to control or overcome tumor-induced Treg activities. New therapies targeting all Foxp $3^{+}$ populations might provide interesting insights into this issue.

We also observed in untreated mice that tumors are infiltrated by $\mathrm{CD} 4^{+}$Teffs and Tregs but relatively few $\mathrm{CD} 8^{+}$Teffs. Tregs are present at a higher ratio in the tumor than in the lymph nodes when compared with $\mathrm{CD}^{+} \mathrm{T}$ cells and at almost a 1:1 ratio compared with $\mathrm{CD}^{+} \mathrm{T}$ cells. It has previously been shown that Tregs can suppress $\mathrm{CD}^{+} \mathrm{T}$ cell effector activity by decreasing IFN- $\gamma$ production $(39,40)$ or cytotoxic activity $(41)$, but to date no studies have correlated infiltration of Foxp $3^{+} \mathrm{T}$ cells into the tumor and $\mathrm{CD}^{+}$ $\mathrm{T}$ cell infiltration and activity. Here we extend the previous studies by showing that $\mathrm{CD} 4^{+} \mathrm{CD} 25^{+}$Tregs can suppress tumor-specific $\mathrm{CD}^{+} \mathrm{T}$ cell responses by reducing their proliferation, IFN- $\gamma$ production, and, most importantly, intratumor accumulation. Previous studies have also shown that antitumor responses can be achieved following intratumor depletion of $\mathrm{CD}^{+} \mathrm{T}$ cells (42) or by systemic depletion of CD $25^{+} \mathrm{T}$ cells (29). Our data confirm the synergistic effect of Treg depletion and Gvax therapy, in terms of both tumor rejection and, more mechanistically, tumor infiltration by $\mathrm{CD}^{+} \mathrm{T}$ cells. Together these data suggest that the tumorinfiltrating $\mathrm{CD} 4{ }^{+} \mathrm{CD} 25^{+}$Tregs exert dominant inhibitory control over infiltrating $\mathrm{CD} 8^{+}$Teffs and that this may contribute to the lack of an effective immune response to tumors.

Gvax/anti-CTLA4 treatment induced a striking augmentation in $\mathrm{CD}^{+}$Teff and $\mathrm{CD} 8^{+}$Teff infiltration and in the percentage of IFN- $\gamma$-producing $\mathrm{T}$ cells within the tumor, resulting in an increase in the intratumoral ratios of both $\mathrm{CD}^{+}$and $\mathrm{CD} 8^{+}$Teffs to Tregs. This may overcome Treg-mediated suppression and tip the balance toward tumor rejection. The impact of CTLA4 blockade on the $\mathrm{CD}^{+}$compartment may be either indirect or direct. Although in vitro studies suggest that CTLA4 blockade has a greater impact on $\mathrm{CD}^{+}$than $\mathrm{CD}^{+} \mathrm{T}$ cell responses (43), effective antitumor responses can be elicited by Gvax/anti-CTLA4 even in the absence of CD $4^{+}$ $\mathrm{T}$ cells (14). The latter finding suggests that CTLA4 blockade may exert an effect by acting directly on $\mathrm{CD}^{+} \mathrm{T}$ cells rather than solely by enhancing $\mathrm{CD}^{+} \mathrm{T}$ cell help. In contrast to the situation within the tumor, combination therapy increased the percentage of Tregs in the lymph nodes, resulting in a reduction in the Teff/Treg ratios. This is also consistent with the increase in frequency of Tregs in lymph nodes upon chronic CTLA4 blockade.

The alterations in the balance of Teffs and Tregs that were induced in the tumor and in the lymph nodes were not demon- strable directly when blood was used as an analyte. This has clear implications for the monitoring of immunotherapy studies in humans and closely parallels the experience gained in such clinical studies. It may well be that tumor-specific $T$ cells do not circulate in large numbers in the blood but rather are resident in the tumor itself or in tumor-draining lymph nodes. Laboratory studies of human lymph node and tumor samples would help to resolve this issue.

Our study suggests a model for the differential impact of combination therapy on the intranodal and intratumoral lymphocyte populations and also provides an explanation for the ineffectiveness of CTLA4 blockade and Gvax as monotherapies for poorly immunogenic tumors. In the absence of any therapy, tumors induce the expansion and accumulation of Tregs in the lymph nodes. In untreated mice, tumors are poorly infiltrated by $\mathrm{CD}^{+}$ Teffs, and the dominant infiltrates are $\mathrm{CD} 4^{+}$Teffs and Tregs. The relatively high numbers of Tregs compared with Teffs maintain a state of hyporesponsiveness to the tumor and prevent its rejection. We propose that CTLA4 blockade removes cell-autonomous restraining mechanisms that inhibit the proliferation of both Treg and Teff compartments. Anti-CTLA4 monotherapy does not result in increased intratumoral $\mathrm{T}$ cell infiltration, and the ratios of $\mathrm{CD}^{+}$and, particularly, $\mathrm{CD}^{+}$Teffs to Tregs remain low within the tumor. However, in the absence of Gvax therapy, there is an accumulation of Tregs within the lymph nodes driven by self antigen. Gvax facilitates efficient priming of tumor-specific Teffs. As a monotherapy, this induces some degree of tumor infiltration and IFN- $\gamma$ production by $\mathrm{CD}^{+}$Teffs. However, intratumoral proliferation will still be under the restraints imposed by CTLA4-mediated signaling. Combination with CTLA4 blockade results in maximal effects on Teff numbers and, most significantly, in Teff/Treg ratios by allowing unrestrained proliferation driven by tumor antigens, resulting in the inversion of the Teff/Treg ratios. The eventual functional outcome of therapeutic intervention will depend on the relative ratio of Teffs to Tregs within the tumor. With combination therapy, the priming induced by Gvax results in a massive increase in Teffs within the tumor, and the inhibitory activities of the Tregs are overwhelmed, resulting in tumor rejection. In the absence of Gvax, an insufficient number of Teffs infiltrate the tumor, and the outcome of CTLA4 blockade still favors Tregs over Teffs, resulting in continued tumor growth.

We believe that the data presented in this article shed light on the mechanisms by which Gvax/anti-CTLA4 induces antitumor activity. Our data are consistent with a cell-autonomous role of CTLA4 on the control of Teffs and Tregs. Blockade of this regulatory pathway has the same effect on both compartments, resulting in their expansion in response to antigenic stimulation. The overall outcome will depend on the local antigenic milieu. Therefore, additional conventional or immune-based interventions aiming at enhancing antigen presentation, such as the coadministration of cellular vaccines capable of selectively expanding tumor-reactive Teffs, are likely to be an essential component of antitumor therapies incorporating anti-CTLA4.

\section{Methods}

Mice. C57BL/6 mice (6-8 weeks old) were purchased from Taconic, maintained in microisolator cages, and treated in accordance with the NIH and American Association of Laboratory Animal Care regulations. Experiments for this study were approved by the Memorial Sloan-Kettering Cancer Center Institutional Animal Care and Use Committee. 
Antibodies. Anti-CTLA4 (clone 9D9), anti-CD25 (clone PC61), and antiCD3 (clone 500A2) were purified from bioreactor supernatants at the Memorial Sloan-Kettering Cancer Center monoclonal hybridoma facility. All other antibodies used for flow cytometry where purchased from eBioscience.

In vitro suppression assays. $\mathrm{CD} 4^{+} \mathrm{CD} 25^{+}$and $\mathrm{CD} 4^{+} \mathrm{CD} 25^{-} \mathrm{T}$ cells were purified from lymph nodes with Miltenyi Biotec magnetic beads (>95\% purity). $50,000 \mathrm{CD} 4^{+} \mathrm{CD} 25^{-}$Teffs were plated in round-bottom 96 -well plates in the presence of 150,000 $\mathrm{T}$ cell-depleted irradiated splenocytes and increasing amounts of $\mathrm{CD}^{+} \mathrm{CD} 25^{+}$Tregs plus $10 \mu \mathrm{g} / \mathrm{ml}$ purified anti-CD3. When used in vitro, anti-CTLA4 (or mouse IgG control) was added at a final concentration of $50 \mu \mathrm{g} / \mathrm{ml}$, which has been described to have maximum effect in in vitro cultures (31). Cells were incubated at $37^{\circ} \mathrm{C}$ for 72 hours and were pulsed with $\left[{ }^{3} \mathrm{H}\right]$ thymidine in the last 8 hours of culture. For suppression of $\mathrm{CD}^{+} \mathrm{T}$ cell responses, $\mathrm{CD} 8{ }^{+} \mathrm{T}$ cells were isolated from lymph nodes of naive mice via negative selection with Miltenyi Biotec magnetic beads (>95\% purity). We incubated $100,000 \mathrm{CD}^{+}$Teffs with different numbers of $\mathrm{CD}^{+}{ }^{+} \mathrm{CD} 25^{+}$Tregs isolated from naive mice or tumor-draining lymph nodes from Gvax/anti-CTLA4-treated mice together with APCs and antiCD3 as described above. Groups were analyzed in quintuplicate, and experiments were repeated at least 3 times. To study the impact of Tregs on tumor-specific $\mathrm{CD}^{+} \mathrm{T}$ cells, mice were challenge with B16/BL6 cells in Matrigel (BD; as described above) and treated with Gvax on days 3, 6, and 9. Fifteen days after tumor challenge, tumors were removed and CD8 ${ }^{+}$TILs were isolated with Miltenyi magnetic beads. We coincubated 50,000 CD8 ${ }^{+}$ TILs with 100,000 DCs and 50,000 tumor cells in the presence or absence of 50,000 CD $4{ }^{+} \mathrm{CD} 25^{+} \mathrm{T}$ cells isolated from naive mice.

Long-term in vivo treatment with anti-CTLA4. Mice were treated for 2 weeks every other day with $100 \mu \mathrm{g}$ anti-CTLA4 or control Ig injected i.p. At the end of the 2 weeks, mice were sacrificed and lymph nodes isolated for purification of Tregs and Teffs. A fraction of the cells was saved for flow cytometric analysis of the cell surface markers CD4 and CD25 and the transcription factor Foxp3 according to the manufacturer's instructions (eBioscience).

Cell lines. The highly tumorigenic and poorly immunogenic cell line B16/ BL6 was used for tumor challenge. B16/BL6-expressing GM-CSF was used for therapy. Both cell lines have been previously described (12)

Tumor challenge and treatment experiments. Mice were injected in the flank i.d. at day 0 with 12,000 B16/BL6 melanoma cells and treated or not on days 3, 6, and 9 with $1 \times 10^{6}$ irradiated (150 Gy) B16/BL6-expressing GM-CSF (Gvax) on the contralateral flank $(n=10), 100 \mu \mathrm{g}$ anti-CTLA4 i.p. $(n=10)$, or a combination of both $(n=10)$. Depletion of CD25+ cells was achieved by i.p. injection of mice with $400 \mu \mathrm{g}$ anti-CD25 monoclonal antibody (clone PC61) 4 days before tumor challenge. Tumor growth and rejection were monitored over time.

Histology and confocal microscopy. Mice were injected in the flank i.d. at day 0 with 12,000 B16/BL6 melanoma cells and treated or not on days 3, 6, and 9 with $1 \times 10^{6}$ irradiated (150 Gy) B16/BL6-expressing GM-CSF (Gvax) together with $100 \mu$ g anti-CTLA4 i.p. At day 15, tumors were dissected and snap-frozen in OCT. Eight-micrometer sections were generated with a cryomicrotome, fixed in acetone, and stained with anti-CD4-FITC, anti-CD8-PE, and anti-Foxp $3^{+}$APCs. Samples were analyzed with a Leica Microsystems inverted confocal microscope with a $\times 20$ water immersion objective.

Isolation of TILs. Mice were injected in the flank i.d. at day 0 with 12,000 B16/BL6 melanoma cells in $200 \mu \mathrm{l}$ of a collagen matrix (Matrigel) that has been shown to facilitate the recovery of TILs from tumor-bearing and tumor-rejecting mice (44). On days 3,6, and 9, mice were injected in the contralateral side with $1 \times 10^{6}$ irradiated (150 Gy) B16/BL6 expressing GM-CSF (Gvax) with or without $100 \mu$ g anti-CTLA4 i.p. Fifteen days after tumor challenge, mice were sacrificed, and TILs were obtained from tumors after a Ficoll gradient was performed to eliminate dead cells. TILs were analyzed by flow cytometry for the expression of CD4, CD8, Foxp $3^{+}$, and IFN- $\gamma$. The same number of cells (based on side-scatter and forward-scatter analyses) was acquired in all samples. The total number of infiltrating $\mathrm{CD}^{+} \mathrm{T}$ cells/gram of tumor was obtained by multiplying the percentage of $\mathrm{CD}^{+}$cells by the total number of lymphocytes obtained from Ficoll and dividing that number by 100 and by the weight of the tumors. For IFN- $\gamma$ production, TILs where incubated for 4 hours with $50 \mathrm{ng} / \mathrm{ml}$ PMA and 500 $\mathrm{ng} / \mathrm{ml}$ ionomycin or overnight with $10 \mu \mathrm{M}$ TRP2 peptide. After restimulation, TILs were stained for flow-cytometric analysis of intracellular cytokines using a Cytofix/Cytoperm kit according to the manufacturer's instructions (BD Biosciences - Pharmingen).

Statistics. Data were analyzed by 2 -tailed Student's $t$ test, and $P<0.05$ was considered statistically significant. Data for tumor rejection was analyzed by Fisher's exact test, and $P<0.05$ was considered statistically significant.

\section{Acknowledgments}

We would like to thank Elisa Cardenas and Katie Wojnoonski for technical assistance and Peter Savage for critical evaluation of the manuscript. Sergio A. Quezada is a Fellow of the Cancer Research Institute, USA. Karl S. Peggs is a Visiting Fellow funded by the Leukaemia Research Fund, London, United Kingdom. James P. Allison is an investigator of the Howard Hughes Medical Institute and holds the David H. Koch Chair in Immunologic Studies at the Memorial Sloan-Kettering Cancer Center.

Received for publication December 21, 2005, and accepted in revised form April 25, 2006.

Address correspondence to: James P. Allison, Howard Hughes Medical Institute, Department of Immunology, Memorial SloanKettering Cancer Center, 1275 York Avenue, New York, New York, USA. Phone: (212) 639-6971; Fax: (212) 717-3212; E-mail: allisonj@mskcc.org.

Sergio A. Quezada and Karl S. Peggs contributed equally to this work.
1. Greenwald, R.J., Freeman, G.J., and Sharpe, A.H. 2005. The B7 family revisited. Annu. Rev. Immunol. 23:515-548.

2. Krummel, M.F., and Allison, J.P. 1996. CTLA-4 engagement inhibits IL-2 accumulation and cell cycle progression upon activation of resting T cells. J. Exp. Med. 183:2533-2540.

3. Brunner, M.C., et al. 1999. CTLA-4-mediated inhibition of early events of $\mathrm{T}$ cell proliferation. J. Immunol. 162:5813-5820.

4. Greenwald, R.J., Boussiotis, V.A., Lorsbach, R.B., Abbas, A.K., and Sharpe, A.H. 2001. CTLA-4 regulates induction of anergy in vivo. Immunity. 14:145-155.
5. Egen, J.G., and Allison, J.P. 2002. Cytotoxic T lymphocyte antigen-4 accumulation in the immunological synapse is regulated by TCR signal strength. Immunity. 16:23-35.

6. Egen, J.G., Kuhns, M.S., and Allison, J.P. 2002 CTLA-4: new insights into its biological function and use in tumor immunotherapy. Nat. Immunol. 3:611-618.

7. Leach, D.R., Krummel, M.F., and Allison, J.P. 1996. Enhancement of antitumor immunity by CTLA-4 blockade. Science. 271:1734-1736.

8. Kwon, E.D., et al. 1997. Manipulation of T cell costimulatory and inhibitory signals for immunotherapy of prostate cancer. Proc. Natl. Acad. Sci. U. S. A.
94:8099-8103.

9. Yang, Y.F., et al. 1997. Enhanced induction of antitumor T-cell responses by cytotoxic T lymphocyteassociated molecule- 4 blockade: the effect is manifested only at the restricted tumor-bearing stages. Cancer Res. 57:4036-4041.

10. Shrikant, P., Khoruts, A., and Mescher, M.F. 1999. CTLA-4 blockade reverses CD8+ T cell tolerance to tumor by a CD4+ T cell- and IL-2-dependent mechanism. Immunity. 11:483-493.

11. Sotomayor, E.M., Borrello, I., Tubb, E., Allison, J.P., and Levitsky, H.I. 1999. In vivo blockade of CTLA-4 enhances the priming of responsive T cells but fails to prevent the induction of tumor 
antigen-specific tolerance. Proc. Natl. Acad. Sci. U. S. A. 96:11476-11481.

12. Van Elsas, A., Hurwitz, A.A., and Allison, J.P. 1999. Combination immunotherapy of B16 melanoma using anti-cytotoxic $\mathrm{T}$ lymphocyte-associated antigen 4 (CTLA-4) and granulocyte/macrophage colony-stimulating factor (GM-CSF)-producing vaccines induces rejection of subcutaneous and metastatic tumors accompanied by autoimmune depigmentation. J. Exp. Med. 190:355-366.

13. Hurwitz, A.A., Yu, T.F., Leach, D.R., and Allison, J.P. 1998. CTLA-4 blockade synergizes with tumorderived granulocyte-macrophage colony-stimulating factor for treatment of an experimental mammary carcinoma. Proc. Natl. Acad. Sci. U. S. A. 95:10067-10071.

14. Van Elsas, A., et al. 2001. Elucidating the autoimmune and antitumor effector mechanisms of a treatment based on cytotoxic $\mathrm{T}$ lymphocyte antigen-4 blockade in combination with a B16 melanoma vaccine: comparison of prophylaxis and therapy. J. Exp. Med. 194:481-489.

15. Dranoff, G., et al. 1993. Vaccination with irradiated tumor cells engineered to secrete murine granulocyte-macrophage colony-stimulating factor stimulates potent, specific, and long-lasting anti-tumor immunity. Proc. Natl. Acad. Sci. U. S. A. 90:3539-3543.

16. Huang, A.Y., et al. 1994. Role of bone marrowderived cells in presenting MHC class I-restricted tumor antigens. Science. 264:961-965.

17. Hodi, F.S., et al. 2003. Biologic activity of cytotoxic $\mathrm{T}$ lymphocyte-associated antigen 4 antibody blockade in previously vaccinated metastatic melanoma and ovarian carcinoma patients. Proc. Natl. Acad. Sci.U.S. A. 100:4712-4717.

18. Ribas, A., et al. 2004. Role of dendritic cell phenotype, determinant spreading, and negative costimulatory blockade in dendritic cell-based melanoma immunotherapy. J. Immunother. 27:354-367.

19. Phan, G.Q., et al. 2003. Cancer regression and autoimmunity induced by cytotoxic $\mathrm{T}$ lymphocyte-associated antigen 4 blockade in patients with metastatic melanoma. Proc. Natl. Acad. Sci. U. S. A. 100:8372-8377.

20. Korman, A., Yellin, M., and Keler, T. 2005. Tumor immunotherapy: preclinical and clinical activity of anti-CTLA4 antibodies. Curr. Opin. Investig. Drugs.
6:582-591.

21. Peggs, K.S., Quezada, S.A., Korman, A.J., and Allison, J.P. 2006. Principles and use of anti-CTLA4 antibody in human cancer immunotherapy. Curr. Opin. Immunol. 8:206-213.

22. Sakaguchi, S. 2004. Naturally arising CD4+ regulatory t cells for immunologic self-tolerance and negative control of immune responses. Annu. Rev. Immunol. 22:531-562.

23. Sakaguchi, S. 2005. Naturally arising Foxp3expressing $\mathrm{CD} 25+\mathrm{CD} 4+$ regulatory $\mathrm{T}$ cells in immunological tolerance to self and non-self. Nat. Immunol. 6:345-352.

24. Graca, L., Cobbold, S.P., and Waldmann, H. 2002. Identification of regulatory $\mathrm{T}$ cells in tolerated allografts. J. Exp. Med. 195:1641-1646.

25. Lee, I., et al. 2005. Recruitment of Foxp3+ T regulatory cells mediating allograft tolerance depends on the CCR4 chemokine receptor. J. Exp. Med. 201:1037-1044.

26. Curiel, T.J., et al. 2004. Specific recruitment of regulatory $T$ cells in ovarian carcinoma fosters immune privilege and predicts reduced survival. Nat. Med. 10:942-949.

27. Ko, K., et al. 2005. Treatment of advanced tumors with agonistic anti-GITR $\mathrm{mAb}$ and its effects on tumor-infiltrating Foxp3+CD25+CD4+ regulatory T cells. J. Exp. Med. 202:885-891.

28. Viguier, M., et al. 2004. Foxp3 expressing CD4+CD25(high) regulatory $\mathrm{T}$ cells are overrepresented in human metastatic melanoma lymph nodes and inhibit the function of infiltrating $\mathrm{T}$ cells. J. Immunol. 173:1444-1453.

29. Sutmuller, R.P., et al. 2001. Synergism of cytotoxic $\mathrm{T}$ lymphocyte-associated antigen 4 blockade and depletion of $\mathrm{CD} 25(+)$ regulatory $\mathrm{T}$ cells in antitumor therapy reveals alternative pathways for suppression of autoreactive cytotoxic T lymphocyte responses. J. Exp. Med. 194:823-832.

30. Read, S., Malmstrom, V., and Powrie, F. 2000. Cytotoxic $\mathrm{T}$ lymphocyte-associated antigen 4 plays an essential role in the function of CD25(+)CD4(+) regulatory cells that control intestinal inflammation. J. Exp. Med. 192:295-302.

31. Takahashi, T., et al. 2000. Immunologic self-tolerance maintained by $\mathrm{CD} 25(+) \mathrm{CD} 4(+)$ regulatory $\mathrm{T}$ cells constitutively expressing cytotoxic T lymphocyte-associated antigen 4. J. Exp. Med. 192:303-310.
32. Kataoka, H., et al. 2005. CD25(+)CD4(+) regulatory $\mathrm{T}$ cells exert in vitro suppressive activity independent of CTLA-4. Int. Immunol. 17:421-427.

33. Tang, Q., et al. 2004. Distinct roles of CTLA-4 and TGF-beta in CD4+CD25+ regulatory $\mathrm{T}$ cell function. Eur. J. Immunol. 34:2996-3005.

34. Maker, A.V., Attia, P., and Rosenberg, S.A. 2005 Analysis of the cellular mechanism of antitumor responses and autoimmunity in patients treated with CTLA-4 blockade. J. Immunol. 175:7746-7754.

35. Thornton, A.M., Piccirillo, C.A., and Shevach, E.M. 2004. Activation requirements for the induction of CD4+CD25+ T cell suppressor function. Eur. J. Immunol. 34:366-376.

36. Keler, T., et al. 2003. Activity and safety of CTLA-4 blockade combined with vaccines in cynomolgus macaques. J. Immunol. 171:6251-6259.

37. Attia, P., et al. 2005. Autoimmunity correlates with tumor regression in patients with metastatic melanoma treated with anti-cytotoxic T-lymphocyte antigen-4. J. Clin. Oncol. 23:6043-6053.

38. Ghiringhelli, F., et al. 2005. Tumor cells convert immature myeloid dendritic cells into TGF-betasecreting cells inducing CD4+CD25+ regulatory $\mathrm{T}$ cell proliferation. J. Exp. Med. 202:919-929.

39. Dittmer, U., et al. 2004. Functional impairment of CD8(+) T cells by regulatory $\mathrm{T}$ cells during persistent retroviral infection. Immunity. 20:293-303.

40. Piccirillo, C.A., and Shevach, E.M. 2001. Cutting edge: control of CD8+ $\mathrm{T}$ cell activation by CD4+CD25+immunoregulatory cells. J. Immunol. 167:1137-1140.

41. Chen, M.L., et al. 2005. Regulatory T cells suppress tumor-specific CD8 T cell cytotoxicity through TGF-beta signals in vivo. Proc. Natl. Acad. Sci. U. S. A. 102:419-424.

42. Yu, P., et al. 2005. Intratumor depletion of CD4+ cells unmasks tumor immunogenicity leading to the rejection of late-stage tumors. J. Exp. Med. 201:779-791.

43. Chambers, C.A., Kuhns, M.S., and Allison, J.P. 1999. Cytotoxic T lymphocyte antigen-4 (CTLA-4) regulates primary and secondary peptide-specific CD4(+) T cell responses. Proc. Natl. Acad. Sci. U. S. A. 96:8603-8608.

44. Kowalczyk, D.W., et al. 2001. A method that allows easy characterization of tumor-infiltrating lymphocytes. J. Immunol. Methods. 253:163-175. 